\title{
Idiopathic Adrenal Hematoma Masquerading as Neoplasm
}

\author{
Kazuki Sasaki Terumasa Yamada Kunihito Gotoh \\ Hirotada Kittaka Hidenori Takahashi Masahiko Yano \\ Hiroaki Ohigashi Osamu Ishikawa \\ Department of Surgery, Osaka Medical Center for Cancer and Cardiovascular \\ Diseases, Osaka, Japan
}

\section{Key Words}

Adrenal hematoma $\cdot$ Adrenal hemorrhage $\cdot$ Incidentaloma

\begin{abstract}
We report herein a case of idiopathic adrenal hematoma. A 59-year-old Japanese man was referred to our hospital for evaluation of a $7.0 \mathrm{~cm}$ mass in the right upper abdominal cavity. The tumor was suspected to originate from either the posterior segment of the liver or the right adrenal gland. His chief complaint was weight loss of $8 \mathrm{~kg}$ over the previous 6 months. He had no past medical history and took no medications, including no anticoagulants. Laboratory data were almost normal except for a slight elevation of PIVKA-II. The origin of the tumor was found to be the adrenal gland, as angiography revealed the blood supply to the mass to derive from the right superior and inferior adrenal arteries. A fine needle biopsy of the lesion was unable to confirm the diagnosis. Open right adrenalectomy was performed. The histopathological findings of the surgical specimen revealed a hematoma with normal adrenal tissue. In the absence of any obvious etiology, the diagnosis was idiopathic adrenal hematoma.
\end{abstract}

\section{Introduction}

Adrenal hemorrhage is a relatively rare condition [1]. It is caused by trauma, stress, sepsis, adrenal tumors, anticoagulation, hemorrhagic disorders and pregnancy. An adrenal hematoma in the absence of any distinct etiology is termed an 'idiopathic adrenal hematoma'. The symptoms vary from subclinical to clinical, such as nausea, abdominal pain, fever and hypotension due to circulatory collapse. It is sometimes found incidentally by imaging examination for another reason. Adrenal tumors associated with hemorrhage primarily include pheochromocytomas, adrenocortical cancers and metastatic lesions from other organs. From a clinical perspective, whether 
the lesion is benign or malignant is an important issue but is difficult to determine prior to surgery. We describe a case of idiopathic adrenal hemorrhage and discuss the surgical indications.

\section{Case Report}

A 59-year-old Japanese man was referred to our hospital for evaluation of a $7.0 \mathrm{~cm}$ mass in the posterior segment of the liver or right adrenal gland in November 2009. This mass was discovered when abdominal ultrasonography was obtained to evaluate a weight loss of $8 \mathrm{~kg}$ from May to November 2009. He had no past medical history and was not taking any anticoagulants. His family history was unremarkable. His height was $166 \mathrm{~cm}$ and his weight was $52.5 \mathrm{~kg}$. His body temperature was $37.0^{\circ} \mathrm{C}$, his blood pressure was $124 / 73 \mathrm{~mm} \mathrm{Hg}$, and his pulse was regular at $67 / \mathrm{min}$. Physical examination revealed nothing remarkable. Complete blood count and biochemical tests were within normal limits. Hormonal examination revealed a slightly elevated metanephrine level $(0.35 \mathrm{mg} / \mathrm{day}$; normal range 0.04-0.19). Tumor marker levels, including serum $\alpha$-fetoprotein, carcinoembryonic antigen and carbohydrate antigen 19-9 were all within normal ranges. Only PIVKA-II was slightly elevated at $53 \mathrm{mAU} / \mathrm{ml}($ normal <37). Hepatitis B antigen and anti-hepatitis C virus antibody were negative.

Abdominal computed tomography (CT) demonstrated a well-demarcated, $7.0 \mathrm{~cm}$ lesion in either the posterior segment of the liver or in the right adrenal gland. The peripheral region was slightly enhanced and the central part was hypovascular, indicating central necrosis (fig. 1a, b). There was no evidence of lymph node enlargement or distant metastasis. On magnetic resonance imaging (MRI), the mass appeared grossly hypointense on gadolinium chelate-enhanced T1-weighted image (fig. 1c) and marginally hyperintense on T2-weighted image (fig. 1d). On abdominal angiography, the mass showed arterial enhancement and a CT filling defect in the right superior and inferior adrenal arteries and arterial portography, respectively (fig. 2). Therefore, the tumor was determined to be of right adrenal origin. 131I-meta-iodo-benzylguanidine (MIBG) scintigraphy demonstrated no aberrant accumulation in the mass, which ruled out the possibility of a pheochromocytoma. A percutaneous fine needle biopsy was performed, but only blood clot was obtained. For the purposes of securing a definite diagnosis and treatment, an open right adrenalectomy was planned for March 2010. The preoperative diagnosis was adrenal tumor.

For surgery the patient was placed in the supine position. Laparotomy was performed through a $\mathrm{J}$-shaped incision. The liver appeared normal. The lesion seemed to be localized to the right adrenal gland. The liver was mobilized by incision of the falciform, triangular and coronary ligaments. The main lesion was covered by the capsule. The mass did not directly invade the liver and could be easily detached. The right adrenal vein and artery were ligated and the mass removed. No drainage tube was placed. Operative time and estimated blood loss were $164 \mathrm{~min}$ and $250 \mathrm{ml}$, respectively.

The resected specimen was $7.0 \times 5.5 \mathrm{~cm}$ in size, and the cut surface of the mass was heterogeneous and dark red and white in appearance (fig. 3 ). On histopathological examination, hematoxylin-eosin staining revealed hemorrhage, necrosis and hemosiderin deposit with normal adrenal tissue. Neoplastic tissue was not observed. In the absence of any obvious etiology, the final pathological diagnosis was idiopathic adrenal hematoma. The patient had an uneventful postoperative course and was discharged home on postoperative day 12. Follow-up was uneventful without complications.

\section{Discussion}

The incidence of adrenal hemorrhage identified at autopsy has been reported to be $1.1 \%$ [1]. The adrenal gland is highly vascular and vulnerable to hemorrhage by nature [2]. Small adrenal branches from the three main adrenal arteries form a subcapsular plexus, and the gland is drained by relatively few venules. For example, stress increases adrenal vascularity and increases adrenal venous pressure due to vasoconstriction, resulting in intraglandular hemorrhage. 
Idiopathic adrenal hematomas are difficult to diagnose prior to surgery. In Japan, Koizumi et al. [3] described 14 cases of idiopathic adrenal hematomas from 1983 to 2010. Of these 14 cases, 13 had been suspected to be adrenal tumors including malignant lesions preoperatively, but were then found to be 'idiopathic adrenal hematomas' on pathological examination after surgery. Only 1 patient was diagnosed with an idiopathic adrenal hematoma without surgery. The adrenal hemorrhage was not suspected to be caused by an adrenal tumor on the basis of CT, hormonal assay and MIBG results, and this mass spontaneously regressed after 2 weeks [4].

Although imaging modalities such as CT and MRI are helpful in detecting adrenal hemorrhage [5], it is difficult to determine whether the hemorrhage is associated with tumors or not. Marti et al. [6] reviewed 133 cases of adrenal hemorrhage with associated masses. Adrenal hemorrhage associated with pheochromocytoma (48\%) was the most frequently observed situation, and the second most frequently observed was a malignant lesion (20\%) such as adrenocortical cancer or metastasis from another organ. Hematomas derived from pseudocysts or adenomas comprised $17 \%$ of all cases. Therefore, even if pheochromocytoma is ruled out by hormonal evaluations and imaging studies, the possibility that the mass may be a malignant lesion remains approximately $50 \%$. Furthermore, the size of adrenal incidentalomas, which are adrenal gland masses discovered serendipitously on imaging, is an important factor in differentiating benign tumors from malignant lesions. Nieman [7] recommended routine surgical resection for adrenal incidentalomas $>4 \mathrm{~cm}$ in diameter without a clear-cut diagnosis.

In our case, metastatic liver tumors, hemorrhaged hepatic cellular carcinoma with central necrosis and adrenal tumor were raised as differential diagnoses at the time of the initial consultation because of ring enhancement with hypovascularity on enhanced CT and a slightly elevated PIVKA-II level. Subsequently, abdominal angiography revealed the origin of the mass to be the right adrenal gland. Although vital signs, laboratory data and MIBG scintigraphy ruled out the possibility of a pheochromocytoma, the possibility of another malignancy could not be completely ruled out. Therefore, surgery was strongly recommended for a definite diagnosis as well as for treatment. The reason for the patient's weight loss and the increase in the PIVKA-II in our case was not clear, but may have been related to exhaustion during the summer season from May until November that kept him from eating sufficiently. In fact, his weight loss ceased after he consulted our hospital, and the PIVKA-II value also normalized prior to surgery.

In conclusion, we report herein a case of idiopathic adrenal hematoma. An accurate diagnosis of idiopathic adrenal hemorrhage is quite difficult to make prior to surgery. Some imaging modalities are useful in generating a differential diagnosis, but if the potential for malignancy is not excluded, surgical resection should be taken into consideration.

\section{Disclosure Statement}

None of the authors has any conflicts of interest with regard to this paper. 


\begin{tabular}{r|l|l|l}
$\begin{array}{r}\text { Case Reports in } \\
\text { Gastroenterology }\end{array}$ & $\begin{array}{l}\text { Case Rep Gastroenterol 2012;6:171-176 } \\
\text { DOI: 10.1159/000338063 }\end{array}$ & $\begin{array}{l}\text { Published online: } \\
\text { April 3, 2012 }\end{array}$ & $\begin{array}{l}\text { @ 2012 S. Karger AG, Basel } \\
\text { ISSN 1662-0631 } \\
\text { www.karger.com/crg }\end{array}$ \\
\hline
\end{tabular}
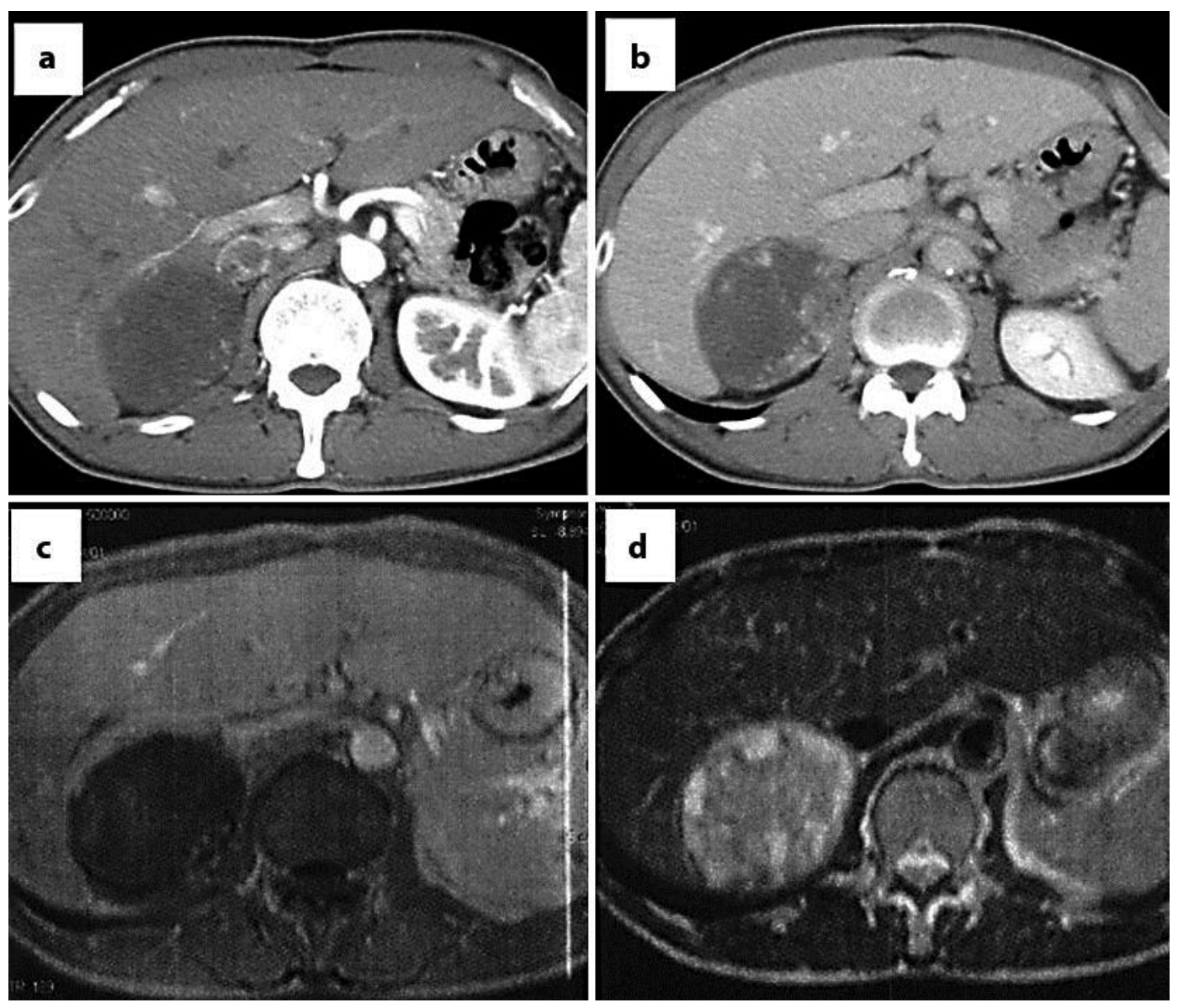

Fig. 1. Abdominal CT and MRI. Abdominal CT showed a $7.0 \mathrm{~cm}$ mass at either the posterior segment of the liver or the right adrenal gland. The peripheral part was heterogeneously enhanced in the arterial phase (a) and the portal phase (b). In gadolinium chelate-enhanced T1-weighted MRI image, the lesion appeared almost hypointense (c). T2-weighted MRI revealed some partially hyperintense rims around the mass (d). 


\begin{tabular}{r|l|l|l}
$\begin{array}{r}\text { Case Reports in } \\
\text { Gastroenterology }\end{array}$ & $\begin{array}{l}\text { Case Rep Gastroenterol 2012;6:171-176 } \\
\text { DOI: } 10.1159 / 000338063\end{array}$ & $\begin{array}{l}\text { Published online: } \\
\text { April 3, 2012 }\end{array}$ & $\begin{array}{l}\text { ○ 2012 S. Karger AG, Basel } \\
\text { ISSN 1662-0631 } \\
\text { www.karger.com/crg }\end{array}$ \\
\hline
\end{tabular}
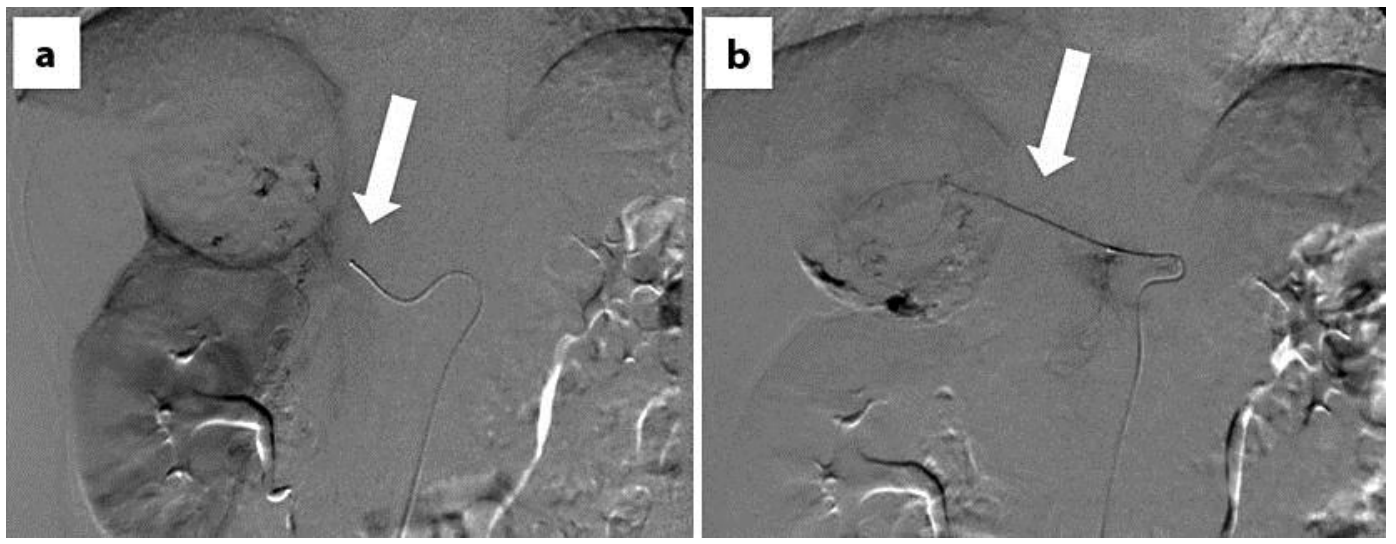

Fig. 2. Abdominal angiography. The feeding vessels included branches from the right inferior adrenal artery (a) and the right superior adrenal artery (b).
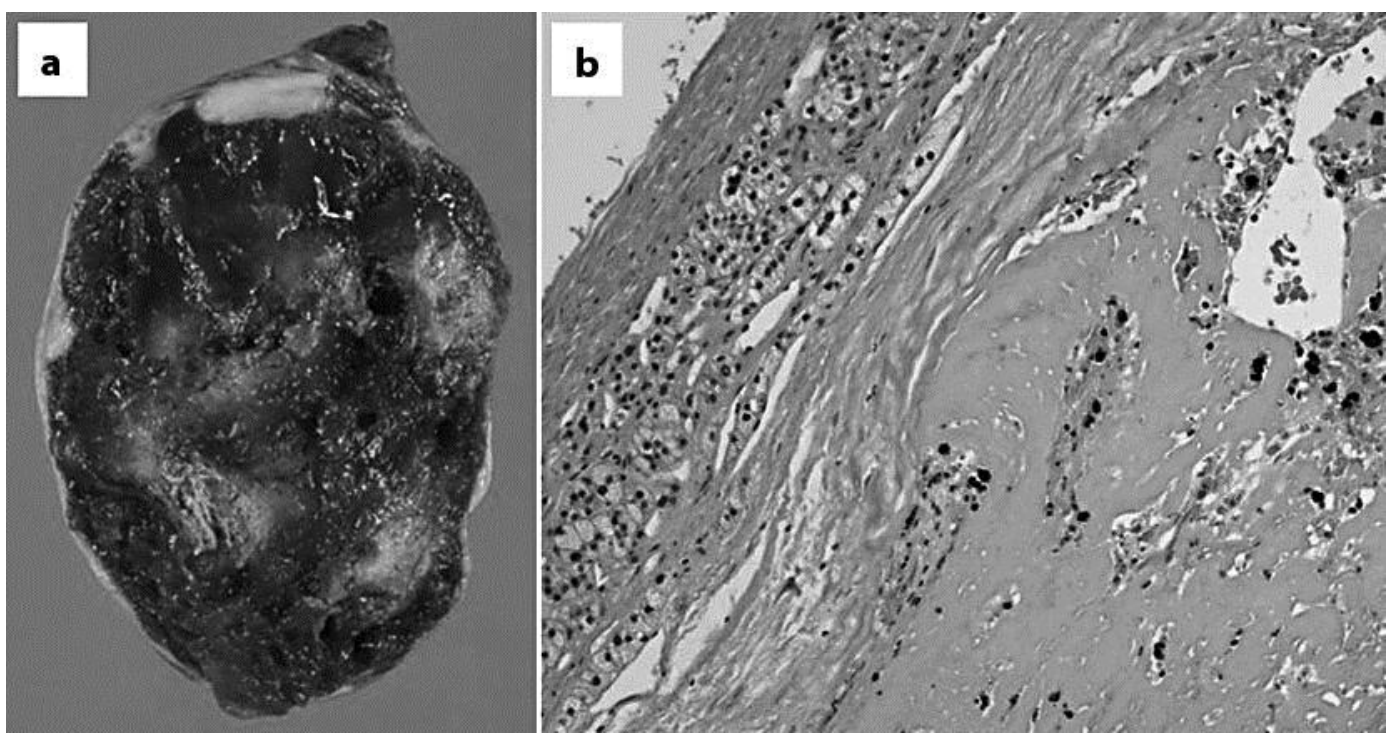

Fig. 3. Gross appearance and histopathology. The cut surface of the resected specimen revealed a well-defined dark red and white solid mass. The weight of the mass was $120 \mathrm{~g}$ (a). It was composed of hemorrhage, necrosis, hemosiderin deposits and normal adrenal tissue without any neoplastic lesions (hematoxylin-eosin stains, $\times 100)(b)$.

\section{References}

1 Xarli VP, Steele AA, Davis PJ, Buescher ES, Rios CN, Garcia-Bunuel R: Adrenal hemorrhage in the adult. Medicine 1978;57:211-221.

2 Rao RH, Vagnucci AH, Amico JA: Bilateral massive adrenal hemorrhage: early recognition and treatment. Ann Intern Med 1989;110:227-235.

3 Koizumi M, Sata N, Sakuma Y, Shimizu A, Hyodo M, Yasuda Y: A case of giant idiopathic adrenal hematoma (in Japanese with English abstract). J Jpn Soc Clin Surg 2010;71:1314-1318.

4 Sato H, Ichino M, Yanaoka M: A case of idiopathic adrenal hemorrhage (in Japanese with English abstract). J Shizuoka Red Cross Hosp 2002;22:93-96. 
-5 Kawashima A, Sandler CM, Ernst RD, Takahashi N, Roubidoux MA, Goldman SM, Fishman EK, Dunnick NR: Imaging of nontraumatic hemorrhage of the adrenal gland. Radiographics 1999;19:949-963.

-6 Marti JL, Millet J, Sosa JA, Roman SA, Carling T, Udelsman R: Spontaneous adrenal hemorrhage with associated masses: etiology and management in 6 cases and a review of 133 reported cases. World J Surg 2012;36:75-82.

7 Nieman LK: Approach to the patient with an adrenal incidentaloma. J Clin Endocrinol Metab 2010;95: 4106-4113. 